\title{
Connection forms of an orthonormal frame field in the Minkowski space
}

\author{
Keziban Orbay \\ Amasya University, Faculty of Education, Amasya, Turkey \\ E-mail address: \\ keziban.orbay@amasya.edu.tr
}

\section{To cite this article:}

Keziban Orbay. Connection Forms of an Orthonormal Frame Field in the Minkowski Space. Pure and Applied Mathematics Journal. Special Issue: Applications of Geometry. Vol. 4, No. 1-2, 2015, pp. 10-13. doi: 10.11648/j.pamj.s.2015040102.13

\begin{abstract}
In this work, connection formulas and forms of an orthonormal frame field in the Minkowski space $I R_{1}^{3}$ were introduced and then the variation of connection forms was studied. In addition, the relation between the matrix of connection forms and the transition matrix of an orthonormal basis of tangent space were established, and an example was illustrated.
\end{abstract}

Keywords: Minkowski Space, One-Form, Connection Forms

\section{Introduction}

It is well-know that Euclidean geometry is a very useful tool in classical mechanics. On the other hand, Riemannian geometry has tremendous amount of applications in general relativity. Therefore, differential geometry has always given rise to new branches of physics [3,7]. Over the years, differential forms have generated a considerable amount of interest not only because they are interesting, but also important as they influenced the research direction both in Euclidean and Lorentzian geometries. Some of the works in this direction are given in $[2,4,6]$ where the authors studied connections forms. In particular, they investigated covariant derivatives of frame elements as connected to this frame and they obtained connection forms and their matrices.

In the spirit of this study, we investigated the connection formulas and forms of an orthonormal frame field in the Minkowski space $I R_{1}^{3}$. This paper is organized as follows: subsequently, we provided the background material concerning the basic concepts and definitions. Then, we study the variation of connection forms. In particular, we establish the relationship between the matrix of connection forms and the transition matrix of an orthonormal basis of the tangent space, and we present an example. In the final section, we summarize our results.

\section{Preliminaries}

We consider the Minkowski 3-space $I R_{1}^{3}$ with the scalar product

$$
\langle\vec{X}, \vec{Y}\rangle=x_{1} y_{1}+x_{2} y_{2}-x_{3} y_{3}
$$

where $\vec{X}=\left(x_{1}, x_{2}, x_{3}\right)$ and $\vec{Y}=\left(y_{1}, y_{2}, y_{3}\right)$ are vectors in $I R_{1}^{3}$. $\vec{X}$ and $\vec{Y}$ are called perpendicular if $\langle\vec{X}, \vec{Y}\rangle=0$. The norm of $\vec{X}$ is defined by $\|\vec{X}\|=\sqrt{|\langle\vec{X}, \vec{X}\rangle|} \cdot \vec{X}$ is called space-like if $\langle\vec{X}, \vec{X}\rangle>0$ or $\vec{X}=\overrightarrow{0}$, time-like if $\langle\vec{X}, \vec{X}\rangle<0$ and light-like(null) if $\langle\vec{X}, \vec{X}\rangle=0$ and $\vec{X} \neq \overrightarrow{0}$ [5]. The cross product of $\vec{X}$ and $\vec{Y}$ is defined by [1]

$$
\vec{X} \Lambda \vec{Y}=\left(x_{3} y_{2}-x_{2} y_{3}, x_{1} y_{3}-x_{3} y_{1}, x_{1} y_{2}-x_{2} y_{1}\right)
$$

Let $\vec{\alpha}: I \rightarrow I R_{1}^{3}, I \subset I R$, be a regular curve in $I R_{1}^{3}$, and consider the tangent vector $\dot{\vec{\alpha}}(s), s \in I \subset I R$. Then in [5],

1) $\vec{\alpha}$ is a space-like curve if $\langle\dot{\vec{\alpha}}(s), \dot{\vec{\alpha}}(s)\rangle>0$,

2) $\vec{\alpha}$ is a time-like curve if $\langle\dot{\vec{\alpha}}(s), \dot{\vec{\alpha}}(s)\rangle<0$,

3) $\vec{\alpha}$ is a null curve if $\langle\dot{\vec{\alpha}}(s), \dot{\vec{\alpha}}(s)\rangle=0$.

Let $\vec{\alpha}(s)$ be a space-like curve of unit speed in $I R_{1}^{3}$ with the natural curvature $\kappa(s)$ and torsion $\tau(s)$. Let us consider the Frenet frame $\{\vec{t}, \vec{n}, \vec{b}\}$ of $\vec{\alpha}(s)$ where $\vec{t}, \vec{n}$ and $\vec{b}$ are the space-like unit tangent vector, time-like unit principal normal vector and space-like unit binormal vector, respectively. Then scalar and cross product of $\vec{t}, \vec{n}$ and $\vec{b}$ are given by

$$
\langle\vec{t}, \vec{t}\rangle=-\langle\vec{n}, \vec{n}\rangle=\langle\vec{b}, \vec{b}\rangle=1,\langle\vec{t}, \vec{n}\rangle=\langle\vec{t}, \vec{b}\rangle=\langle\vec{n}, \vec{b}\rangle=0,
$$




$$
\vec{t} \Lambda \vec{n}=-\vec{b}, \vec{n} \Lambda \vec{b}=-\vec{t}, \vec{b} \Lambda \vec{t}=\vec{n} .
$$

Finally, Frenet formulas are given by [8]

$$
\dot{\vec{t}}=\kappa(s) \vec{n}, \quad \dot{\vec{n}}=\kappa(s) \vec{t}+\tau(s) \vec{b}, \quad \dot{\vec{b}}=\tau(s) \vec{n} .
$$

\section{Main Results}

Let $\left\{E_{1}, E_{2}, E_{3}\right\}$ be an orthonormal frame field in the Minkowski space in which $E_{3}$ is a time-like vector. Consider a tangent vector $v_{p} \in T_{p}\left(I R_{1}^{3}\right)$ at any $P \in I R_{1}^{3}$. Let $\mathrm{D}$ be the Levi Civita connection on $I R_{1}^{3}$. Inspired by Frenet formulas, we can consider the covariant derivative of vector fields $E_{i}$, $1 \leq i \leq 3$, with respect to the tangent vector $v_{p}$ as connected to this frame field. Since $D_{v_{p}} E_{i} \in T_{p}\left(I R_{1}^{3}\right)$, and $\left\{E_{1}(p), E_{2}(p), E_{3}(p)\right\}$ is an orthonormal basis of tangent space $T_{p}\left(I R_{1}^{3}\right)$ with $w_{i j}\left(v_{p}\right) \in I R$, the covariant derivative of $E_{i}, 1 \leq i \leq 3$, with respect to $v_{p}$ can be written by

$$
\text { (3.1) } D_{v_{p}} E_{i}=\sum_{j=1}^{3} w_{i j}\left(v_{p}\right) E_{j}(p)
$$

where

$$
\text { (3.2) } w_{i j}\left(v_{p}\right)=\left\langle D_{v_{p}} E_{i}, E_{j}(p)\right\rangle \varepsilon_{j} \text { with } \varepsilon_{j}= \begin{cases}1 & , j \neq 3 \\ -1, & j=3\end{cases}
$$

From a geometric point of view, this equation extracts the number $w_{i j}\left(v_{p}\right)$ that is component of the variation of vector $E_{i}$ with respect to $E_{j}(p)$ where the tangent vector $v_{p}$ is the velocity vector along a curve.

Let $v_{p}, u_{p} \in T_{p}\left(I R_{1}^{3}\right)$ and let $a, b \in I R$. Since

$$
w_{i j}\left(a v_{p}+b u_{p}\right)=a w_{i j}\left(v_{p}\right)+b w_{i j}\left(u_{p}\right)
$$

the transformation $\left(w_{i j}\right)_{p}: T_{p}\left(I R_{1}^{3}\right) \rightarrow I R$, defined by

$$
\left(w_{i j}\right)_{p}\left(v_{p}\right)=w_{i j}\left(v_{p}\right)
$$

is linear. Thus $w_{i j}$ corresponds to a linear transformation from the tangent space $T_{p}\left(I R_{1}^{3}\right)$ to IR for all $\mathrm{P}$ in the Minkowski space $I R_{1}^{3}$. In this case, note that $\left(w_{i j}\right)_{p}$ is an element of the cotangent space $T_{p}^{*}\left(I R_{1}^{3}\right)$, that is, a one-form in $I R_{1}^{3}$.

Theorem 3.1: One-forms $w_{i j}$ of the orthonormal frame field $\left\{E_{1}, E_{2}, E_{3}\right\}$ in which $E_{3}$ is a time-like vector are given by $w_{i j}=-\varepsilon_{i} \varepsilon_{j} w_{j i}, 1 \leq i, j \leq 3$.

Proof: Since $\left\langle E_{i}, E_{j}\right\rangle: I R_{1}^{3} \rightarrow I R$ is a constant function, we have $v_{p}\left\langle\left\langle E_{i}, E_{j}\right\rangle\right]=0$ for all $v_{p} \in T_{p}\left(I R_{1}^{3}\right)$. On the other hand, the equation

$$
v_{p}\left[\left\langle E_{i}, E_{j}\right\rangle\right]=\left\langle D_{v_{p}} E_{i}, E_{j}(p)\right\rangle+\left\langle E_{i}(p), D_{v_{p}} E_{j}\right\rangle
$$

Implies that

$$
\left\langle D_{v_{p}} E_{i}, E_{j}(p)\right\rangle=-\left\langle E_{i}(p), D_{v_{p}} E_{j}\right\rangle
$$

Thus

$$
\begin{aligned}
w_{i j}\left(v_{p}\right) & =\varepsilon_{j}\left\langle D_{v_{p}} E_{i}, E_{j}(p)\right\rangle \\
& =-\varepsilon_{j}\left\langle D_{v_{p}} E_{j}, E_{i}(p)\right\rangle \\
& =-\varepsilon_{j} \varepsilon_{i}\left(\varepsilon_{i}\left\langle D_{v_{p}} E_{j}, E_{i}(p)\right\rangle\right) \\
& =-\varepsilon_{j} \varepsilon_{i} w_{j i}\left(v_{p}\right) .
\end{aligned}
$$

Since the equation is true for all $v_{p} \in T_{p}\left(I R_{1}^{3}\right)$, we obtain

$$
w_{i j}=-\varepsilon_{j} \varepsilon_{i} w_{j i}
$$

Definition 3.1: One-forms $w_{i j}$ are called connection forms of the orthonormal frame field $\left\{E_{1}, E_{2}, E_{3}\right\}$.

Definition 3.2: The equation given in (3.1) is called connection formulas of the orthonormal frame field $\left\{E_{1}, E_{2}, E_{3}\right\}$.

Using Theorem 3.1, we can obtain the matrix $W=\left[w_{i j}\right]_{3 \times 3}$ of one-forms as

$$
W=\left[\begin{array}{ccc}
0 & w_{12} & w_{13} \\
-w_{12} & 0 & w_{23} \\
w_{13} & w_{23} & 0
\end{array}\right] .
$$

Note that $\mathrm{W}$ is a skew-adjoint matrix in the sense that $W^{t}=-\varepsilon W \varepsilon$, where $\mathcal{E}$ is the signature matrix given by

$$
\varepsilon=\left[\begin{array}{ccc}
1 & 0 & 0 \\
0 & 1 & 0 \\
0 & 0 & -1
\end{array}\right] .
$$

Let $\left\{\frac{\partial}{\partial x_{1}}, \frac{\partial}{\partial x_{2}}, \frac{\partial}{\partial x_{3}}\right\}$ be the natural frame field in the Minkowski space $I R_{1}^{3}$ in which $\frac{\partial}{\partial x_{3}}$ is a time-like vector. Then the vector $E_{i}, 1 \leq i \leq 3$, can be written as

$$
\text { (3.3) } E_{i}=\sum_{j=1}^{3} a_{i j} \frac{\partial}{\partial x_{j}}
$$

where $\quad a_{i j}: I R_{1}^{3} \rightarrow I R \quad$ is a differentiable function. Let $A=\left[a_{i j}\right]_{3 \times 3}$ be the transition matrix between the orthonormal bases $\left\{E_{1}(p), E_{2}(p), E_{3}(p)\right\} \quad$ and $\left\{\frac{\partial}{\partial x_{1}}(p), \frac{\partial}{\partial x_{2}}(p), \frac{\partial}{\partial x_{3}}(p)\right\}$ of the tangent space $T_{p}\left(I R_{1}^{3}\right)$. The equation given in (3.3) can be written as 


$$
\text { (3.4) }\left[\begin{array}{l}
E_{1} \\
E_{2} \\
E_{3}
\end{array}\right]=A\left[\begin{array}{c}
\frac{\partial}{\partial x_{1}} \\
\frac{\partial}{\partial x_{2}} \\
\frac{\partial}{\partial x_{3}}
\end{array}\right] \text {. }
$$

Note that $\mathrm{A}$ is an orthogonal matrix, that is, $A^{-1}=\varepsilon A^{t} \varepsilon$. Now we are ready to state the relation between the matrices A and W.

Theorem 3.2: The skew-adjoint matrix $\mathrm{W}$ and the orthogonal matrix A satisfy $W=d A \cdot A^{-1}$.

Proof: Let $v_{p}$ be an element of $T_{p}\left(I R_{1}^{3}\right)$. Then,

$$
\begin{aligned}
D_{v_{p}} E_{i} & =D_{v_{p}}\left(\sum_{k=1}^{3} a_{i k} \frac{\partial}{\partial x_{k}}\right) \\
& =\sum_{k=1}^{3} D_{v_{p}}\left(a_{i k} \frac{\partial}{\partial x_{k}}\right) \\
=\sum_{k=1}^{3}\left(v_{p}\left[a_{i k}\right]\right. & \left.\frac{\partial}{\partial x_{k}}(p)+a_{i k}(p) D_{v_{p}}\left[\frac{\partial}{\partial x_{k}}\right]\right) \\
& =\sum_{k=1}^{3} v_{p}\left[a_{i k}\right] \frac{\partial}{\partial x_{k}}(p)
\end{aligned}
$$

and therefore

$$
\begin{aligned}
w_{i j}\left(v_{p}\right) & =\left\langle D_{v_{p}} E_{i}, E_{j}(p)\right) \varepsilon_{j} \\
& =\left\langle\sum_{k=1}^{3} v_{p}\left[a_{i k}\right] \frac{\partial}{\partial x_{k}}(p), \sum_{l=1}^{3} a_{j l} \frac{\partial}{\partial x_{l}}(p)\right\rangle \varepsilon_{j} \\
& =\left[v_{p}\left[a_{i 1}\right] a_{j 1}(p)+v_{p}\left[a_{i 2}\right] a_{j 2}(p)-v_{p}\left[a_{i 3}\right] a_{j 3}(p)\right] \varepsilon_{j} \\
& =\left[\left(d a_{11}\right)\left(v_{p}\right) a_{j 1}(p)+\left(d a_{22}\right)\left(v_{p}\right) a_{j 2}(p)-\left(d a_{13}\right)\left(v_{p}\right) a_{j 3}(p)\right] \varepsilon_{j} \\
& =\left[d a_{11} a_{j 1}+d a_{22} a_{j 2}-d a_{33} a_{j 3}\right] \varepsilon_{j}\left(v_{p}\right) .
\end{aligned}
$$

Since this equation is correct for all $v_{p} \in T_{p}\left(I R_{1}^{3}\right)$, we obtain

$$
\varepsilon_{j} w_{i j}=d a_{i 1} a_{j 1}+d a_{i 2} a_{j 2}-d a_{i 3} a_{j 3} .
$$

Finally, the above equation along with the identity $A^{-1}=\varepsilon A^{t} \varepsilon$ imply that $W=d A . A^{-1}$.

Example: Let $(r, \theta, z)$ be the usual cylindrical coordinates in $I R_{1}^{3}$ as indicated in Figure 1. There the coordinate functions are well defined and an inverse mapping exists given by

$$
x=r \cos \theta, y=r \sin \theta, z=z .
$$

Consider the natural frame field $\left\{\frac{\partial}{\partial x}, \frac{\partial}{\partial y}, \frac{\partial}{\partial z}\right\}$ in $I R_{1}^{3}$. The cylindrical frame field $\left\{\frac{\partial}{\partial r}, \frac{\partial}{\partial \theta}, \frac{\partial}{\partial z}\right\}$ is given by

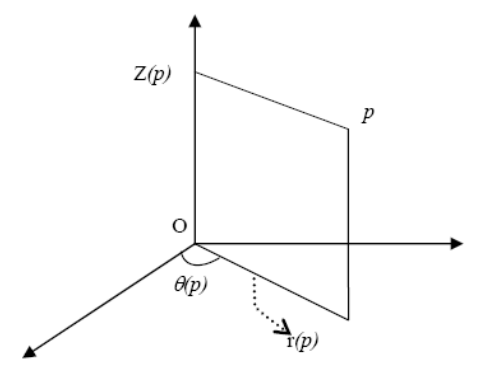

Figure 1. The Cylindrical Coordinate System

$$
\begin{gathered}
\frac{\partial}{\partial r}=\cos \theta \frac{\partial}{\partial x}+\sin \theta \frac{\partial}{\partial y}, \\
\frac{\partial}{\partial \theta}=-r \sin \theta \frac{\partial}{\partial x}+r \cos \theta \frac{\partial}{\partial y}, \\
\frac{\partial}{\partial z}=\frac{\partial}{\partial z} .
\end{gathered}
$$

It follows from the above equations the that the transition matrix $A$ is given by

$$
A=\left[\begin{array}{ccc}
\cos \theta & \sin \theta & 0 \\
-r \sin \theta & r \cos \theta & 0 \\
0 & 0 & 1
\end{array}\right]
$$

Finally, applying Theorem 3.2 we obtain the matrix of connection forms of the cylindrical frame as

$$
\begin{gathered}
W=d A A^{-1} \\
W=\left[\begin{array}{ccc}
-\sin \theta d \theta & \cos \theta d \theta & 0 \\
-r \cos \theta d \theta-\sin \theta d r & -r \sin \theta d \theta+\cos \theta d r & 0 \\
0 & 0 & 0
\end{array}\right]\left[\begin{array}{ccc}
\cos \theta & -r^{-1} \sin \theta & 0 \\
\sin \theta & r^{-1} \cos \theta & 0 \\
0 & 0 & 1
\end{array}\right] \\
W=\left[\begin{array}{ccc}
0 & r^{-1} d \theta & 0 \\
-r d \theta & r^{-1} d r & 0 \\
0 & 0 & 0
\end{array}\right] .
\end{gathered}
$$

\section{Conclusions}

In this paper, we studied the connection forms of an orthonormal frame in the Minkowski space $I R_{1}^{3}$. In contrast to $I R^{3}$, we observed that $w_{i j}$, the component of $E_{i}$ in the direction of $E_{j}(p)$ along a curve with velocity vector $v_{p}$, depends on indices; however, the this dependence does not change the relation between the skew-adjoint matrix $W$ and the orthogonal matrix $A$. We believe that our results will provide a base for further studies, in particular for connection forms in the Minkowski space. 


\section{References}

[1] Akutagawa, K. and Nishikawa S. The Gauss Map and Spacelike Surfaces with Prescribed Mean Curvature in Minkowski 3-space. Tohoku Math. J., 42(2), 1990.

[2] Darling RWR. Differential Forms and Connections, Cambridge University Press, 1994.

[3] Kalimuthu, S. A Brief History of the Fifth Euclidean Postulate and Two New Results. The General Sci. J., 2009, www.wbabin.net/physics/kalimuthu9.pdf.

[4] Morita, S., Nagase, T. and Nomizu, K. Geometry of Differential Forms (Translations of Mathematical Monoqraphs, Vol.201). Amer. Math. Soc., 2001.

[5] O’Neill, B. Semi-Riemannian Geometry with Applications to Relativity. Academic Press, 1983.

[6] O'Neill, B. Elementary Differential Geometry, Revised Second Edition, Academic Press, 2006.

[7] Waner, S. Introduction to Differential Geometry and General Relativity, Hofstra University, 2005.

[8] Woestijne, V.D.I. Minimal Surfaces in the 3-dimensional Minkowski Space, World Scientific Press. Singapore, 1990. 